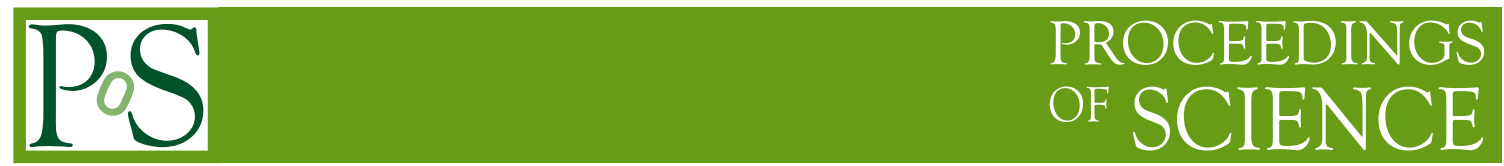

\title{
One-loop finite corrections to seesaw neutrino masses
}

\section{Diego ARISTIZABAL}

IFPA, Dep. AGO, Universite de Liege, Bat B5 Sart Tilman B-4000 Liege 1, Belgium

E-mail: daristizabaleulg.ac.be

In the standard seesaw model, finite corrections to the neutrino mass matrix arise from one-loop self-energy diagrams mediated by heavy neutrinos. We discuss the impact that these corrections may have on the different entries of the tree-level effective neutrino mass matrix, paying special attention to their dependence with the seesaw model parameters. We also briefly comment on the implications these corrections might have on low-energy neutrino observables.

The 2011 Europhysics Conference on High Energy Physics-HEP 2011, July 21-27, 2011 Grenoble, Rhône-Alpes France 


\section{Motivation}

Among the plethora of models for Majorana neutrino masses present in the literature the standard seesaw [1] is certainly the most popular mechanism for neutrino mass generation. In this model, by extending the standard model Lagrangian with three fermionic electroweak singlets, the five dimensional effective operator $L L H H$ is realized via the exchange of the new states. The smallness of the light neutrino masses is determined by the suppression induced by the scale of lepton number violation which is assumed to be large $\mathscr{O}\left(\Lambda_{\mathrm{GUT}}\right)$.

The seesaw parameter space depends upon 18 "coordinates": 6 phases and 12 real parameters. Low-energy data implies-in principle -9 constraints, provided the absolute light neutrino mass scale and the Dirac and Majorana CP violating phases are measured. Given the mismatch between the number of parameters and observables a unique region consistent with data [2] can not be fixed. The analysis of the available portions in parameter space is thus based on scans which in turn rely on parametrizations of the seesaw. All these parametrizations are based on the tree-level effective mass matrix, as the one-loop order corrections are assumed to be negligible. This however might not be the case if the corrections are finite $[3,4,5]$.

\section{One-loop finite corrections}

The fermionic electroweak singlets $N_{R}$ induce new interactions that, in the basis in which the matrix of charged lepton Yukawa couplings and the singlet mass matrix $\boldsymbol{M}_{\boldsymbol{R}}$ are diagonal, are described by the following Lagrangian

$$
-\mathscr{L}=-i \bar{N}_{R_{i}} \gamma_{\mu} \partial^{\mu} N_{R_{i}}+\tilde{\phi}^{\dagger} \bar{N}_{R_{i}} \lambda_{i j} \ell_{L j}+\frac{1}{2} \bar{N}_{R_{i}} C M_{R_{i}} \bar{N}_{R}^{T}+\text { h.c. }
$$

Here $\phi^{T}=\left(\phi^{+} \phi^{0}\right)$ is the Higgs electroweak doublet, $\ell_{L}$ are the lepton $S U(2)$ doublets, $C$ is the charge conjugation operator and $\boldsymbol{\lambda}$ is a Yukawa matrix in flavor space. In the seesaw limit that is to say, $M_{R} \gg v$ (with $v \simeq 174 \mathrm{GeV}$ ) the effective neutrino mass matrix can be written according to

$$
\boldsymbol{m}_{\boldsymbol{v}}^{\text {(tree) }}=-v^{2} \boldsymbol{\lambda}^{T} \hat{\boldsymbol{M}}_{\boldsymbol{R}}{ }^{-1} \boldsymbol{\lambda} .
$$

Finite corrections to the above matrix arise from the one-loop self-energy diagrams shown in figure 1 and are given by 1

$$
\boldsymbol{m}_{\boldsymbol{v}}^{(1-\text { loop })}=v^{2} \lambda^{T} \hat{\boldsymbol{M}}_{\boldsymbol{R}}{ }^{-1}\left\{\frac{g^{2}}{64 \pi^{2} M_{W}^{2}}\left[m_{h}^{2} \ln \left(\frac{\hat{\boldsymbol{M}}_{\boldsymbol{R}}^{2}}{m_{h}^{2}}\right)+3 M_{Z}^{2} \ln \left(\frac{\hat{\boldsymbol{M}}_{\boldsymbol{R}}^{2}}{M_{Z}^{2}}\right)\right]\right\} \boldsymbol{\lambda} .
$$

Notice that this correction is not suppressed with respect to the tree-level result by additional factors of $v \boldsymbol{\lambda} \boldsymbol{M}_{\boldsymbol{R}}{ }^{-1}$. Thus, it is expected to be smaller than the tree-level mass term solely by a factor of order $\left(16 \pi^{2}\right)^{-1} \ln \left(M_{R} / M_{Z}\right)$, implying it might have sizable effects.

We evaluate the importance of the correction in (2.3) by using the Casas-Ibarra parametrization [6]:

$$
\lambda=\frac{\sqrt{\hat{M}_{R}} \boldsymbol{R} \sqrt{\hat{\boldsymbol{m}}_{v}} U^{\dagger}}{v}
$$

\footnotetext{
${ }^{1}$ For details see ref. [3, 4] or the appendix in ref. [5].
} 


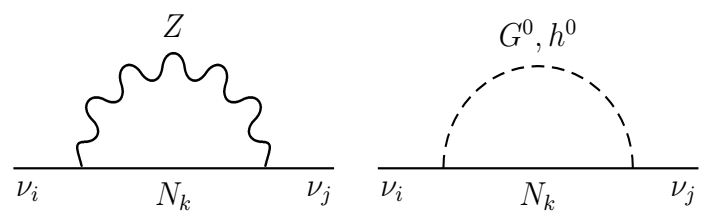

Figure 1: Self-energy diagrams accounting for $\delta \mathbf{M}_{\mathbf{L}}$
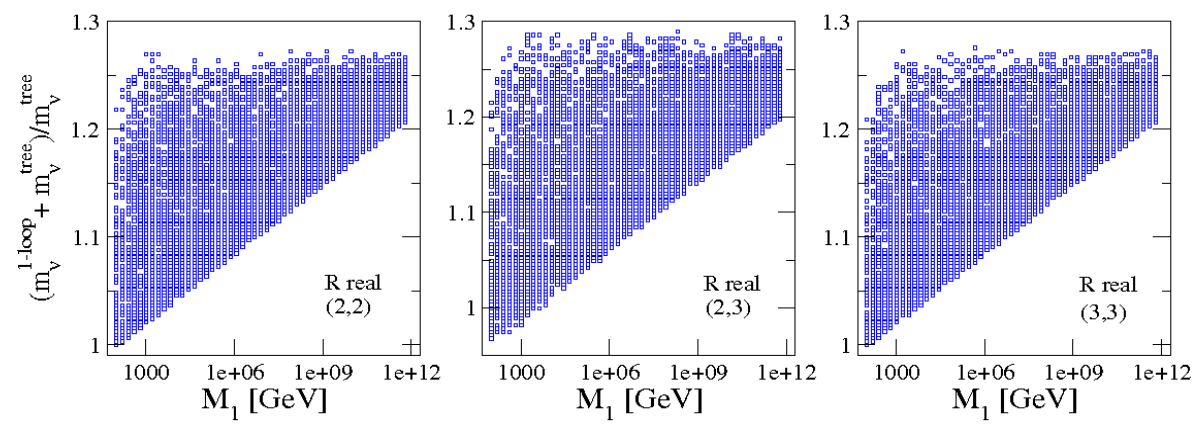

Figure 2: Relevance of the finite one-loop correction for the 22, 23 and 33 elements of the neutrino mass matrix in the case of a real $\boldsymbol{R}$ matrix. The parameter $M_{1}$ corresponds to the lightest electroweak singlet $N_{1}$.

where $\boldsymbol{R}$ is a general complex orthogonal matrix, and scanning the parameter space assuming a normal hierarchical spectrum and a real $\boldsymbol{R}$. The results for the 22, 23 and 33 elements of the mass matrix are displayed in figure 2.

An analysis of how the finite one-loop corrections may affect for example the neutrino mixing angles can be carried out by assuming a well motivated mixing scheme as an input. This has been done in ref. [5] (including also a study of the neutrino mass spectrum) where it has been shown that even in conservative scenarios the effects can be sizable. In conclusion, due to their relevance we argue these corrections must be taken into account in the study of the seesaw parameter space.

\section{Conclusions}

We studied the relevance of the finite one-loop corrections to seesaw neutrino masses. We have found that the corrections can be sizable and may affect the effective light neutrino mass matrix entries, thus implying they can have an impact on the determination of the low-energy neutrino observables (neutrino masses and mixing angles). Our findings therefore prove these corrections should be included in any analysis of the seesaw parameter space.

\section{References}

[1] P. Minkowski, Phys. Lett. B 67421 (1977); T. Yanagida, in Proc. of Workshop on Unified Theory and Baryon number in the Universe, eds. O. Sawada and A. Sugamoto, KEK, Tsukuba, (1979) p.95; M. Gell-Mann, P. Ramond and R. Slansky, in Supergravity, eds P. van Niewenhuizen and D. Z. Freedman (North Holland, Amsterdam 1980) p.315; P. Ramond, Sanibel talk, retroprinted as hep-ph/9809459; S. L. Glashow, inQuarks and Leptons, Cargèse lectures, eds M. Lévy, (Plenum, 1980, New York) p. 
707; R. N. Mohapatra and G. Senjanović, Phys. Rev. Lett. 44, 912 (1980); J. Schechter and J. W. F. Valle, Phys. Rev. D 22 (1980) 2227; Phys. Rev. D 25 (1982) 774.

[2] T. Schwetz, M. Tortola, J. W. F. Valle, New J. Phys. 13, 109401 (2011). [arXiv:1108.1376 [hep-ph]].

[3] A. Pilaftsis, Z. Phys. C 55, 275 (1992) [hep-ph/9901206].

[4] W. Grimus, L. Lavoura, Phys. Lett. B546, 86-95 (2002). [hep-ph/0207229].

[5] D. Aristizabal Sierra, C. E. Yaguna, JHEP 1108, 013 (2011). [arXiv:1106.3587 [hep-ph]].

[6] J. A. Casas, A. Ibarra, Nucl. Phys. B618, 171-204 (2001). [hep-ph/0103065]. 